\title{
Pensamiento de diseño e innovación
}

María Ledesma es doctora en Diseño por la UBA. Actualmente se desempeña como Profesora Titular Regular en la materia Comunicación en la Carrera de Diseño Gráfico en la Universidad de Buenos Aires; Profesora en el Doctorado y en la Maestría en Diseño de la misma universidad; es también Profesora de Teoría y Práctica del Diseño en la Universidad Nacional del Litoral. Integra Comisiones de Doctorado en la FADU-UBA, la FADU UNL y la UNER y dirige la Maestría en Estrategia y Gestión en el Área del Diseño de la UNNOBA. Como profesora invitada ha impartido seminarios y conferencias en universidades y centro educativos de Argentina, Uruguay, Chile, Brasil y Colombia. Es autora, entre otros, de 'Diseño Gráfico, una voz pública', co autora con Arfuch y Chaves de 'Diseño y Comunicación. Teoría y enfoques críticos’ y con López de 'Comunicación para diseñadores', además de numerosos artículos en revistas nacionales y extranjeras. <mariadelvalle.ledesma@gmail.com>
Resumen En la primera parte el artículo ubica al pensamiento del diseño en relación a la ciencia, la tecnología y la innovación, para demostrar que la innovación es constitutiva del diseño a pesar de no estar incluido en la teoría clásica de la innovación. En la segunda parte, a la luz de la experiencia vivida en Argentina, plantea, analiza y describe la diferencia entre los ambientes innovativos de los países con mayor desarrollo tecnológico y los países latinoamericanos. Se caracterizarán diferentes modelos innovativos desarrollados en Argentina y se particularizará una visión del diseño basada en el desarrollo y la inclusión. La conclusión plantea la necesidad de replantear los límites del diseño y llevar esta cuestión a su enseñanza.

Palabras claves Pensamiento proyectual, teoría de la innovación, entornos innovativos, recurso intelectual estratégico.

\section{Design Thinking and Innovation}

Abstract In the first part of this Article, it is claimed that Desgin Thinking is clearly related to Science, Technology and Innovation. It is said that Innovation is a fundamental aspect of Design, in spite of not being included in the traditional theories that defines the concept of Innovation. In the second part of the text, and as a result of the Argentinean experience, it is analyzed and described the difference among Innovation in Developed Countries and the LatinAmerican ones. The Article will described different Innovation Models developed in Argentina and we will discuss how Design can include Development and Social Inclusion. The final idea of this Argumentation will argue how importance is to review our definition of Design, broading it, and start teaching Design based on this redefinition.

Keywords Project thinking, Theory of innovation, Innovative environments, Strategic intellectual resource. 
Parto de la premisa que mucho antes de que la innovación apareciera teorizada en el horizonte de la economía contemporánea, el diseño, cuyo pensamiento es el pensamiento proyectual (no el racional) ya era innovativo. El diseño es en la innovación porque su cualidad es la de la transformación. A esa cualidad de transformación le corresponden un tipo de pensamiento proyectual (diferente al racional) caracterizado por la incertidumbre, la búsqueda y resolución de problemas, la presencia de soluciones diversas, el poder de lo aleatorio, la valoración del contexto y sobre todo, por la capacidad de crear mundos.

Son estas características las que resitúan el pensamiento de diseño en la constelación de saberes contemporáneos: frente a años de 'olvido' en los que se hablaba de los productos diseñados y de su acción en el mundo pero no del pensamiento que los generaba, el diseño ha pasado a ser el modelo de pensamiento en el que economistas o pedagogos buscan referencias sobre un nuevo modo de actuar en el mundo.

Hoy el 'design thinking' está de moda. Desde que Leif Ötsman, con el equipo de Donald Schon, revalorizara los puntos de contacto de la teoría del diseño con la filosofía pragmatista en 2005, el concepto comenzó a expandirse en distintos ámbitos y propone como método de innovación "un enfoque que utiliza la sensibilidad del diseñador y sus métodos de resolución de problemas para satisfacer las necesidades de las personas de un modo tecnológicamente factible y comercialmente viable"(Brown, 2008).

Pero esto que está siendo 'descubierto' es una característica propia del diseño. Dicho de manera mucho más enfática: de todas las disciplinas que proyectan (que prefiguran mundos económicos, sociales, culturales de carácter natural o virtual) las disciplinas del diseño son las únicas que reivindican para sí el carácter proyectual, el carácter de inventar un mundo que funcione, que sea habitable; son las disciplinas del diseño que, concebidas como la acción de generar el hábitat humano, tienen un pensamiento en el que -desde el inicio-se privilegian los rasgos proyectuales del pensamiento respecto de la razón concebida en sentido clásico (un objeto abstracto separado del contexto.)

Desde ese punto de vista, la innovación le es propia y característica. Para las demás disciplinas, la innovación es una necesidad coyuntural, un agregado o en el peor de los casos (la peor literatura sobre los emprendedores) una actitud, un rasgo de carácter. Para el diseño es constitutiva: hacer aparecer algo que no está. Es por eso que en el pensamiento de diseño no tiene estructuras intrínsecas propios de la ciencia, la moral y el arte sino que en él conviven elementos cognitivos, prácticos y estéticos junto a un lugar claro en la producción de bienes materiales. Con Fussler podemos decir que el diseño significa aquel lugar en el cual el arte y la técnica (y por ello, el pensamiento valorativo y el científico) se solapan nuevamente con el fin de allanarle el camino a una nueva cultura (Fussler: 1999, 15) 
1 Ver Pabellón de Seda.

2 Esta es la definición que da Schumpeter a principios del siglo XX: 'la función de los emprendedores es la de reformar o revolucionar las formas de producir poniendo en uso una innovación o, más en general, una posibilidad tecnológica aún no probada de producir una mercancía nueva o de producir una ya conocida en una forma nueva: abriendo una nueva fuente de abastecimiento de materias primas o un nuevo mercado, reorganizando la empresa, etc' (Schumpeter, 1978: 132).
Quizás los desarrollos de Neri Oxman constituyan uno de los ejemplos más fuertes en esa dirección, tanto que su obra merece la misma atención en Museos de Ciencias como en Museos de Artes o Espacios de Diseño ${ }^{1}$.

Sin embargo, sería ingenuo pensar innovación y diseño desde un plano tan general ignorando las vicisitudes de ambos conceptos y su vinculación a la producción capitalista.

La innovación en sentido amplio es un motor del desarrollo capitalista y el concepto ya está en germen en Adam Smith o en David Ricardo. A comienzos del siglo XX, entra en la literatura económica de la mano de Schumpeter quien también desarrolla el concepto de 'emprendedor' ${ }^{2} \mathrm{y}$ desde entonces, ha experimentado una evolución que lo ha llevado desde la innovación de productos hasta innovación en servicio o innovación en procesos productivos o en gestión de organizaciones. Paralelamente, los modelos de innovación se han complejizado pasando desde las concepciones ingenuas que suponían que había una relación directa entre inversión en investigación y desarrollo innovativo hasta la consideración de los procesos de difusión (diferenciación entre invención e innovación) y la importancia de la interacción empresarial.

Si analizamos los ejemplos clásicos de la literatura de la innovación empresarial, dos cosas llaman la atención: en primer lugar la cantidad de ejemplos en los que la innovación es obra del diseño y en segundo lugar, la ausencia de cualquier mención al diseño como disciplina. Este movimiento de afirmación/negación queda claro con los siguientes casos:

1. a mediados de la década de los 90, los japoneses Nonaka y Takeuchi tratando de explicar las razones del crecimiento de las compañías japonesas, llegaron a la conclusión que este éxito se debía a 'la capacidad de una compañía para generar nuevos conocimientos, diseminarlos entre los miembros de la organización y materializarlos en productos, servicios y sistemas' y aseguraban que 'La creación de conocimiento organizacional es la clave del proceso peculiar a través del cual estas firmas innovan', decían. (Nonaka y Takeudi, 1999, 1). El libro es ya un clásico y es ampliamente citado sobre todo por sus conceptualizaciones respecto del ciclo del conocimiento tácito al conocimiento explícito; respecto al tema que nos ocupa interesa hacer hincapié en un aspecto que los autores no consideran a pesar de su evidencia: todos los ejemplos que analizan son ejemplos de diseño. El primero es el análisis del proceso de creación en 1978 del Honda Civic: así nos cuentan que el punto de partida fue el planteo de una misión (crear un auto que no se parezca en nada a los anteriores de la compañía y que sea económico pero no barato); el siguiente implicó la traducción de la misión en un concepto (evolución automotriz) y el tercero, el hallazgo de una metáfora capaz de expresar el concepto. Para resolverlo, fue necesario plantear una serie de preguntas (si el automóvil fuera un organismo, ¿cómo debería evolucionar?) y sus respuestas (hombre 
al máximo, máquina al mínimo) dieron lugar al hallazgo de la metáfora: una esfera (auto corto y alto), creando un concepto que contradecía las convenciones del diseño automotriz vigente.

De esta historia, los autores extraen tres características que están en la base de su teorización sobre la innovación en la empresa: el uso de la metáfora y la analogía para expresar lo inexpresable; la diseminación del conocimiento y la constatación que el nuevo conocimiento nace de la bruma de la ambigüedad y la redundancia. Como se ve, la base de todo una teoría del conocimiento. Nonaka y Tadeuchi fundamentan sus conclusiones en las diferencias de pensamiento entre Oriente y Occidente pero pasan por alto un aspecto esencial: ese modo de pensar también existe en Occidente, sólo que no es el que se enseña en las escuelas ni en las academias, es el pensamiento del diseño.

2. Howard Stevenson de la Universidad de Harvard al teorizar el desplazamiento del concepto de innovación desde el producto hasta la organización- recurre al ejemplo de Mc Donald. (Castillo, 1999) Así Stevenson muestra que McDonald's no ha inventado la hamburguesa si no que ha innovado en el modo de gestionarla; ahora bien, aunque Stevenson no lo diga, ofrecer comida sencilla a gran velocidad supone diseño de herramientas para el proceso y el diseño de espacios con -particulares formas de habitar y trabajar para llevarlo a cabo; el reemplazo del lavavajillas por servilletas y bolsas de papel, es también una decisión de diseño que pone en evidencia que, muchas veces, no se trata de producir algo nuevo sino reutilizar lo antiguo. Pero el ejemplo de McDonald's tiene una segunda parte: la de su valor como empresa icónica del modo de vida americano que ha elevado la acción de comer hamburguesas a rangos insospechados. Esta acción requirió de un aceitado proceso de comunicación visual capaz de generar identificación, adhesión y reconocimiento, a través del logotipo, los colores de la marca, los rituales del servicio y el conjunto de acciones que lo individualizan entre las demás cadenas.

El ejemplo es bueno para mostrar dos aspectos de la innovación realizada por el diseño: el primero que se da en el orden de lo material (la innovación en el proceso productivo, en la disposición de las maquinarias); el segundo que se refiere a la creación de intangibles, valores que contribuyeron a la expansión mundial de la cadena.

Desde este punto de vista, el diseño constituye un aspecto central en la cadena de valor de una empresa u organización que juega de diferente modo según el tipo de cadena, de empresa o de intervención. De manera general, puede intervenir en la generación de productos, en la generación de estrategias de distribución, de consumo.

Tanto el caso del Honda Civic como el de McDonald's teorizados desde concepciones diferentes de la empresa y el emprende- 
dorismo muestran lo que he llamado 'movimiento de afirmación/ negación del diseño': si bien el diseño tiene un modo de ser innovativo, la teoría de la innovación no lo ha tenido en cuenta entre sus aspectos conceptuales.

\section{II}

Como toda práctica social, la del diseño es una práctica situada, que es de una u otra manera según, el tejido ideológico- político en el que se ubique. En realidades como la Argentina y Latinoamérica, profundamente marcadas por la distancia respecto del avance tecnológico de los países desarrollados y por ende, la dificultad para el desarrollo de la competitividad ¿qué condiciones hay para la innovación?

Es clásico considerar que los entornos innovativos se constituyen por la conjunción de buenas universidades, emprendedorismo, financiación, talento, redes sociales-profesionales y calidad de vida. Sillicon Valley es el mayor ejemplo de esto.

Sin embargo, el caso Argentina sirve de contraejemplo para mostrar el papel que juegan la carencia y la crisis en la generación modos de innovación propios en un ambiente tecnológico muy diferente al de los países desarrollados. En contra de lo que podría pensarse, no se trata de una diferencia sólo cuantitativa (menos índices de innovación) sino cualitativa (diferentes índices de innovación).

Pablo Bianchi sostiene en todos los productos de diseño argentino puede reconocerse una característica que define el particular modo de innovar de la región. Pablo Bianchi habla de 'ingenio' que utiliza y resignifica los procesos tecnológicos disponibles para resolver las dificultades del sistema productivo nacional. Son estrategias que buscan racionalizar el uso de los recursos materiales disponibles (Bianchi, 2012). Esta característica propia tuvo su modo de expresión particular durante última década.

La crisis argentina del 2001 desatada por la caída de la convertibilidad monetaria que durante diez años profundizó la destrucción y derrumbe de numerosos sectores productivos, puso en suspenso la hegemonía de los sistemas clásicos de diseño ligados a la mercadotecnia y el desarrollo de la imagen institucional y favoreció la visibilidad de otras modalidades de diseño alejadas del producto y de la empresa tradicional. La respuesta del diseño argentino a la crisis rompió con esquemas cerrados, reproductores de modelos únicos y amplió la noción de contexto de manera que los diseñadores pusieron en diálogo lo global y lo local. Ese pensamiento 'glocal' permitió revalorizar las actividades productivas en pequeña escala, valorizar las artesanías y ensayar una cartografía del diseño diferente a la sancionada por los grandes estudios.

El proceso no tuvo, como suele decirse epicentro en Buenos Aires, sino que reconoce una gran diversidad que va desde las economías urbanas (Buenos Aires, Rosario, Córdoba, Mendoza) hasta las economías basadas en recursos naturales (minerales, forestales, energéticos) pasando por 
aquellas cuyo eje es el turismo y los servicios en general o la combinación de dos o más de ellas en un mismo territorio.

El tipo de respuesta ha sido diversa pero pueden tipificarse en dos grandes grupos:

1. Para describir el primer modo, cito in extenso a Alan Neumarkt quien plantea que "el ingenio creativo de nuestra sociedad logró, en un año, sustituir el sesenta por ciento de todas la importaciones de materias primas y productos industriales; reemplazar los stocks de mercancías importadas por otras de producción nacional; comenzar lentamente un ciclo exportador; generar nuevos productos e instalar la idea de "producto argentino". No sólo los plazos de todo este gran proceso de sustitución han sido muy breves; apenas nos acercamos a los dos años; sino que todo ha sido realizado sin un plan previo y sin crédito. Sin un sistema financiero que lo soporte. Sin ningún tipo de crédito. No deben existir antecedentes de escala comparables, tal vez debiéramos remitirnos a la crisis norteamericana de 1929. El Diseño es hoy la herramienta inicial de todo este proceso y debido a las decenas de proyectos en marcha, donde el Diseño de Moda y el Diseño de Productos son actores principales, creo, que tenemos suficientes casos para hablar de una morfología de la sustitución". (Neumarkt, 2003) Se dio así una explosión de emprendimientos de diseño alternativos que cubren un amplio espectro y modificaron los perfiles urbanos de numerosos barrios en las principales ciudades argentinas: moda, producción de objetos de uso, reapropiación de tecnologías. Citaré dos casos que ilustran la totalidad: hace dos años la Editorial Eterna Cadencia produjo 'el libro que no puede esperar', escrito con una tinta especial que se borra si el libro no se lee en el plazo de tres meses; el año pasado, la valija inteligente argentina se comercializa en USA. Queda claro que fue un fenómeno espontáneo, no planificado ni apoyado por ningún organismo pero con la fuerza suficiente para instalar nuevas formas de producción y consumo.

2. Como ya se dijo, la crisis argentina del 2001 también puso en suspenso la hegemonía de los sistemas clásicos de diseño. Este hecho abrió un espacio para que se hicieran evidentes ideas que se venían perfilando acerca de la capacidad de la inteligencia proyectual de dar respuestas que excedan al producto y se inserten en la trama social, dinamizando sus espacios, sus empresas, su gente. A partir de allí se vio la necesidad de acercar a los distintos sectores de la economía desde los secundarios hasta los quintarios entre otros (Cooper, R y Layard, R, 2003) los instrumentos del diseño, concebido como un recurso estratégico de las sociedades contemporáneas que incide en la obtención de materiales, procedimientos, tecnologías, distribución, almacenamiento, cualidades, percepción 
y atribución de valor. Este último aspecto es remarcable: la concepción del diseño no se centra solo en los productos sino también en los procesos así como el concepto de innovación que subyace no sólo tiene en cuenta la innovación productos sino sobre todo a la gestión de la innovación tal como aparece en el manual de Bogotá, una adecuación local del manual de Oslo.

Esta visión del diseño como recurso intelectual estratégico con fuerte impacto en el desarrollo y calidad de la cadena productiva, de servicios y culturales, da en Argentina (como en toda Latinoamérica) a diferentes líneas de intervención destinadas o bien a la inclusión social o bien al desarrollo regional y han sido tomadas tanto por el Estado como por comunidades independientes con el objetivo de volver más eficientes algunas propuestas destinadas a grupos sociales con débil inserción en el tejido social. Surgen así, por ejemplo, 'corredores productivos' con características diversas. En algunos casos, consolidan y desarrollan sectores productivos medios -la ruta del vino, los corredores turísticos- y en otros, ligan unidades productivas en economías de subsistencia para lograr un enriquecimiento de todo el proceso productivo a través de la cooperación y la solidaridad. Queda claro que el lugar del diseño en estos casos no pasa por el producto sino por articular los diferentes agentes y las diferentes acciones que intervienen en el proceso.

Quiero mencionar acá las experiencias llevadas adelante por el INTI (Instituto Nacional de Tecnología dependiente del Ministerio de Industria, Ciencia y Tecnología), el CMD (Centro Metropolitano de Diseño de Buenos Aires) y a personas como Beatriz Galán, Paulina Becerra, Raquel Ariza, Analía Cirvini, Pablo Bianchi -entre otros- cuyas producciones apuntan en ese sentido. Todos parten de diferentes contextos situacionales (vinculados a procesos pedagógicos, a temas salud y a emprendimientos -empresariales/ territoriales), diagnostican problemas y formulan alternativas de solución, sustentadas en diversos modelos pero con un punto de partida común: reconocer el valor innovativo del diseño en el tejido productivo local.

perada en diciembre del 2001 y contó con el aporte de diseñadores tanto para el diseño de sus productos como para el planteo de su imagen de marca. La importancia de Brukman se ha reflejado en numerosas producciones nacionales e internacionales; me refiero acá a la exhibición 'Pasos para huir del trabajo al hacer', realizada en el museo Ludwig de Colonia (Alemania) bajo la curaduría de los artistas berlineses Andreas Siekmann y Alice Creischer. Las trabajadoras de Brukman por decisión de asamblea aceptaron la propuesta de diseñar y bordar diez trajes que narraran la historia de la toma.

\section{Conclusión}

Si bien, como he intentado mostrar, innovación y diseño son absolutamente compatibles, los rasgos y procesos propios que la innovación contemporánea tanto en los países desarrollados como en los nuestros, nos ponen frente a preguntas que cuestionan los propios límites del diseño.

Las cúpulas orgánicas de Neri Oxman hechas a partir de gusanos de seda y los trajes de la fábrica recuperada Brukman ${ }^{3}$ nviados a la exposición de arte de Colonia (Alemania) son dos puntas del mismo proceso: la transformación del pensamiento del diseño. Hay quienes hablan de crisis del proyecto, hay quienes arriesgan una postproyectualidad o un postdiseño, 
hay quienes discuten la calidad de diseño poniendo en juego categorías que no están hechas para pensar en las producciones colectivas y hay quienes insisten en que la sensibilidad operativa del diseño se podrá aplicar a cualquier tipo de actividad (Manzini, 2013).

Dado que considero enfáticamente que lo arquitectónico y lo diseñado existen sólo como resultado de una producción específicamente cultural e histórica, no cuesta nada recordar que sólo bajo ciertas condiciones sociales, se han construido edificios y artefactos, vestidos y comunicaciones con propósitos que pueden considerarse 'arquitectónicos' o 'diseñados'. Y por lo tanto, es lógico suponer que estén mutando.

La discusión que se abre nos obliga a considerar esas preguntas, sobre todo pensando en los modos de enseñanza del diseño. En Argentina, este nuevo modo de considerar el diseño aún no ha sido trasladado a los planes de estudio -por la inercia propia de la burocracia universitaria-; sin embargo ha entrado en el discurso universitario y desde allí se replica en lo que sería un 'ecosistema innovador', al estilo latinoamericano.

\section{Referencias}

BECERRA, P y otros. Diseñar para la inclusión, Buenos Aires, Ed FADU, 2011.

BIANCHI, P. Diseño Argentino. Ingenio e Identidad en Pul no7 Buenos Aires, 2012.

BROWN, T. Desgin Thinking, Harvard Business Review América Latina, 2008. Disponible https://emprendedoresupa.files.wordpress.com/2010/08/p02_brown-design-thinking.pdf. CASTILLO, A. Estado del arte en la enseñanza del emprendimiento Chile, INTEC, 1999. CORSANI, A y LAZZARATO, M. Capitalismo cognitivo, propiedad intelectual y creación colectiva. Madrid, Traficantes de Sueños, 2010.

FUSSLER, V. Diseño. Madrid, Cerezo, 1999.

MANZZINI, E. Entrevista realizada en el no 20 de la Revista 'Abre el ojo', Madrid, 2013.

MEDINA, C. Ciencia y Tecnología: un enfoque administrativo, México, UAM, Ascapotzalco, 1994.

NEUMARKT, A. Diseño: el plato principal. Un análisis del futuro del Diseño desde Buenos Aires, Argentina en “Cuadernos de Diseño”, Madrid, Instituto Europeo de Diseño (AAVV), 2003. NONAKA, I y TADEUCHI, H. La organización creadora de conocimiento. Como las compañías japonesas crean la innovación', México, Oxford University Press, 1999.

SCHUMPETER, J. Teoría del desenvolvimiento económico, México, FCE, 1978. 